\title{
ON FINITE $p$-GROUPS WITH ISOMORPHIC MAXIMAL SUBGROUPS
}

\author{
PETER Z. HERMANN
}

(Received 30 May 1988; revised 3 October 1988)

Communicated by $\mathbf{H}$. Lausch

\begin{abstract}
Finite p-groups with all of their maximal subgroups isomorphic are studied by means of the coclass. All such groups of coclass 1 and 2 are determined, while those of coclass 3 are shown to have order at most $p^{13}$. A general bound for the order is given as a function of $p$ and the coclass only.
\end{abstract}

1980 Mathematics subject classification (Amer. Math. Soc.) (1985 Revision): 20 D 15.

The aim of the present paper is the investigation of finite $p$-groups with all maximal subgroups isomorphic, by means of their coclass. It will be shown that the order of such a $p$-group is bounded by a function of $p$ and the coclass only. For the particular case of coclass at most 2, a complete characterization of the above groups is provided, while for coclass 3 we give a big improvement for the bound on the group order, at least compared to the one resulting from the general estimate. The methods used throughout are perfectly elementary and are mainly based on Lemma 1 below.

Notation. For a finite $p$-group $X$ we denote by $1=Z_{0}(X)<Z_{1}(X)<\cdots$ and by $X=\gamma_{1}(X)>\gamma_{2}(X)>\cdots$ the terms of the ascending and descending central series of $X$, respectively. (Higher) commutators of two subgroups $A$ and $B$ will be denoted by $[A, B ; 1]=[A, B],[A, B ; 2]=[[A, B ; 1], B]$, etc. Let $[A, B ; 0]$ simply stand for $A$. The nilpotency class of $X$ is abbreviated

This research was supported by the Hungarian National Foundation for Scientific Research, grant number 1813.

(C) 1990 Australian Mathematical Society 0263-6115/90 \$A2.00+0.00 
by $\operatorname{cl}(X)$; if $X$ is of order $p^{n}$ and of class $k$, the coclass of $X$ is $\operatorname{cocl}(X)=$ $n-k$. We will use the standard notation $\Phi(X)$, or merely $\Phi$, for the Frattini subgroup of $X$. A group is said to be an $(M I)$-group if all of its maximal subgroups are isomorphic.

When determining the automorphism group of a finite $p$-group $P$, one can investigate the induced linear group $A$ on the Frattini factor $P / \Phi$ as a first step. In doing so, information about the orbits of $A$ on the set of all hyperplanes can be obtained by knowing the isomorphism classes of the maximal subgroups in $P$. (MI)-groups just provide the situation when that reduction fails.

In turn, if the automorphism group of $P$ induces a transitive linear group on the hyperplanes (or, equivalently, on the lines) of $P / \Phi$ then $P$ is $(M I)$. In particular, the finitely generated (relatively) free groups in any variety defined by the identities $x^{p^{m}}=1, \operatorname{cl}(X) \leq k$ are certainly $(M I)$. There are nonfree groups with induced transitive linear group as the latter can be prescribed (compare with Bryant and Kovács [1]). In addition, for $p=2$ some of the Suzuki 2-groups are also known to be $(M I)$ (see Higman [4]). (MI)-groups with nontransitive induced linear groups appear in Theorem 2.

Since every finite $p$-group is a homomorphic image of some relatively free group, $(M I)$-groups of arbitrarily high class, coclass and solubility length exist. It can be seen for the same reason that the class $(M I)-p$ is not closed under taking homomorphic images. Simple examples yield the same for subgroups and direct products. However, for all known $(M I)$-group $P, P / Z(P)$ is also $(M I)$. Were that true for all $(M I)$-groups, the big bound $f(c)$ of the estimate in Theorem 1 could be replaced by $g(c)=3 c$. That is certainly fulfilled by any group with an induced transitive linear group.

LEMMA 1. Let $G$ be a finite p-group. If $G$ is $(M I)$ and $Z_{n}(G) \neq G$, then $Z_{n}(G) \leq \Phi(G)$.

Proof. Suppose the contrary and choose a subgroup $R$ in $G$ to be of minimal order with $G=Z_{n}(G) \cdot R$. Let $R \leq M$ and $|G: M|=p$. By assumption, we can find a subgroup $R_{1}$ in $R$ such that $Z_{n}(G) \cap R \leq R_{1}$ and $\left|R: R_{1}\right|=p$. Then $Z_{n}(G) \cdot R_{1}$ is a maximal subgroup in $G$, hence it is isomorphic to $M$. Thus we can write $M=T K$ with $T \triangleleft M, K \leq M$, $[T, M ; n]=1$ and $|K|=\left|R_{1}\right|$. Clearly $Z_{n}(G) \cdot M=G$.

ClaIM. $[T, G ; k] \leq Z_{n-k}(G) \cdot[T, M ; k]$ for all $k$.

Our claim can be proved by induction on $k$; it holds trivially for $k=0$. Assume that $[T, G ; i] \leq Z_{n-i}(G) \cdot[T, M ; i]$, then $[T, G ; i+1] \leq\left[Z_{n-i}(G)\right.$. $[T, M ; i], G] \leq Z_{n-i-1}(G) \cdot\left[[T, M ; i], Z_{n}(G) \cdot M\right] \leq Z_{n-i-1}(G) \cdot\langle[T, M ; i+$ 1], [[T,M;i], $\left.\left.Z_{n}(G)\right]^{m}: m \in M\right\rangle \leq Z_{n-i-1}(G) \cdot\left\langle[T, M ; i+1],\left[\gamma_{i+1}(G), Z_{n}(G)\right]\right\rangle$; 
by the well-known assertion due to P. Hall, $\left[\gamma_{i+1}(G), Z_{n}(G)\right] \leq Z_{n-i-1}(G)$ (see [2] or [5, p. 265]), and therefore one can conclude that $[T, G ; i+1] \leq$ $Z_{n-i-1}(G) \cdot[T, M ; i+1]$, as required. For $k=n$ we have $[T, G ; n] \leq Z_{0}(G)$. $[T, M ; n]=1$, that is $T \leq Z_{n}(G)$, thus $G=Z_{n}(G) \cdot M=Z_{n}(G) \cdot T K=$ $Z_{n}(G) \cdot K$ with $|K|=\left|R_{1}\right|<|R|$, a contradiction.

We need some simple facts about $(M I)$-groups; their proofs are left to the reader. $p^{3}$.

LEMMA 2. If $G$ is a nonabelian finite p-group and it is $(M I)$, then $\left|Z_{2}(G)\right| \geq$

LEMMA 3. Let $G$ be a finite p-group. If $G$ is $(M I)$ and $\operatorname{cocl}(G)=1$ (that is, $G$ is of maximal class), then $p^{2} \leq|G| \leq p^{3}$.

COROLlary 1. G is (MI) of coclass 1 if and only if it is

(i) elementary Abelian of order $p^{2}$, or

(ii) nonabelian of order $p^{3}$ and of exponent $p$ with $p>2$, or

(iii) the quaternion group of order 8.

LeMma 4. Assume that $\left|Z_{i}(G): Z_{i-1}(G)\right|=p$ for all $t<i \leq s$ (for some $t<s)$. If $G$ is a p-group, $Z_{t}(G) \leq N \leq Z_{s}(G)$ and $N \triangleleft G$, then $N=Z_{j}(G)$ for some $j$.

LEMMA 5. If $M_{1}$ and $M_{2}$ are different maximal subgroups of a finite $p$ group $G$ and $Z_{s}\left(M_{1}\right), Z_{s}\left(M_{2}\right)$ are both contained in $Z_{t}(G)$ (for some $s$ and $t$ ), then $Z_{s+1}\left(M_{1}\right) \cap Z_{s+1}\left(M_{2}\right) \leq Z_{t+1}(G)$.

Lemma 6. Assume that the finite p-group $G$ is $(M I)$ and $\operatorname{cocl}(G)=c$. If $M_{1}$ and $M_{2}$ are different maximal subgroups in $G$ with $Z_{s}\left(M_{i}\right) \leq Z_{t}(G)(i=1,2)$, then $Z_{s+1}\left(M_{i}\right) \leq Z_{t+2 c+1}(G)(i=1,2)$. If $|G|>p^{2 s+3 c-2}$, then in addition $Z_{s+1}\left(M_{i}\right) \leq Z_{t+2 c-1}(G)$.

LEMMA 7. There is no finite (MI)-group $G$ with $Z(G)=Z(M)$ of order $p$ for all maximal subgroups $M$.

THEOREM 1. Let $G$ be a finite p-group of coclass $c$. If $G$ is $(M I)$, then $|G| \leq p^{f(c)}$, where $f(c)=(2 c+1)^{c+1}+c+2$.

Proof. Let $M$ be any maximal subgroup of $G$. Denote $Z_{j}(G)$ simply by $Z_{j}$; then by Lemmas 1 and 6 we get from the trivial inclusion $Z_{0}(M) \leq Z_{0}$ that $Z_{i} \leq Z_{i}(M) \leq Z_{i(2 c+1)}$ for all $i$. We proceed by induction on $|G|=p^{n}$. 
CASE 1. There exists a minimal $i$ in the set $\left\{1,(2 c+1),(2 c+1)^{2}, \ldots\right.$, $\left.(2 c+1)^{c-1}\right\}$ such that $\left|Z_{i(2 c+1)}: Z_{i}\right|=p^{2 c i}$.

We have $Z_{i}(M)=Z_{i+s}$ (for some $0 \leq s \leq 2 c i$ ) by Lemma 4. (Thanks to the $(M I)$-property, $s$ is independent of $M$.) Let $\left|Z_{i}\right|=p^{i+t}\left(\geq p^{i+1}\right.$ by Lemma 2$)$, then $\left|Z_{i+s}\right|=p^{i+t+s}, \bar{G}=G / Z_{i+s}$ is of order $p^{n-i-t-s}$, it is $(M I)$ and $\operatorname{cocl}(\bar{G})=$ $c-t<c$; therefore $|G|=p^{i+t+s} \cdot|\bar{G}| \leq p^{i+t+s+f(c-t)} \leq p^{i(2 c+1)+t+f(c-t)} \leq$ $p^{(2 c+1)^{c}+t+f(c-t)} \leq p^{f(c)}$ by induction.

CASE 2. $\left|Z_{j(2 c+1)}: Z_{j}\right|>p^{2 c j}$ for all $j \in\left\{1,(2 c+1), \ldots,(2 c+1)^{c-1}\right\}$.

Now $G / Z_{(2 c+1)^{c}}$ is of maximal class, hence $T=Z_{(2 c+1)^{c}}(M)$ is either independent of $M$, or $|G: T| \leq p$. In any case, $|G: T| \leq p^{3}$ by Lemma 3 , thus $|G| \leq p^{3} \cdot|T| \leq p^{3} \cdot\left|Z_{(2 c+1)^{c+1}}\right|=p^{3+(2 c+1)^{c+1}+c-1}=p^{f(c)}$ since $T \leq Z_{(2 c+1)^{c+1}}$.

For the case $c=1$ the above formula gives $p^{f(1)}=p^{12}$ only (instead of the correct value of $p^{3}$ ). The situation becomes even worse for $c>1$, when $f(c)$ will turn out to be far too big. To give some refinement for $c \leq 3$, we firstly deal with the case $c=2$ by proving

THEOREM 2. Assume that the finite $p$-group $G$ is $(M I)$ and that $\operatorname{cocl}(G)=2$; then $G$ is isomorphic to one of the groups listed below:

(iv) $\left\langle a, b: a^{9}=b^{9}=[a, b]^{3}=[a, b, a, a]=[a, b, b, b]=1\right.$,

$$
\left.[a, b, a]=b^{3},[a, b, b]=a^{3}\right\rangle
$$

(v) $\left\langle a, b: a^{9}=b^{9}=[a, b]^{3}=[a, b, a, a]=[a, b, b, b]=1\right.$,

$$
\left.[a, b, a]=b^{6},[a, b, b]=a^{3}\right)
$$

(vi) $\left\langle a, b: a^{p^{2}}=b^{p^{2}}=[a, b]^{p}=[a, b, a, a]=[a, b, b, b]=1\right.$,

$$
\left.[a, b, a]=b^{p},[a, b, b]=a^{p m}\right\rangle
$$

(for $p \geq 5$ and $m$ the smallest quadratic nonresidue $\bmod p$ );

(vii) $\left\langle a, b: a^{p^{2}}=b^{p^{2}}=[a, b]^{p}=[a, b, a, a]=[a, b, b, b]=1\right.$,

$$
\left.[a, b, a]=b^{p},[a, b, b]=a^{p g} b^{p}\right\rangle
$$

(for $p \geq 5,1 \leq g \leq p-1$ and $4 g+1$ any quadratic nonresidue $\bmod p$, which gives $(p-1) / 2$ groups of this type);

(viii) $\left\langle a, b: a^{p}=b^{p}=[a, b]^{p}=[a, b, a]^{p}=[a, b, b]^{p}=[a, b, a, a]\right.$

$$
=[a, b, a, b]=[a, b, b, a]=[a, b, b, b]=1\rangle \quad(\text { for } p \geq 5) \text {; }
$$


(ix) $\left\langle a, b: a^{p^{2}}=b^{p^{2}}=[a, b]^{p}=[a, b, a, b]=[a, b, b, a]=1\right.$,

$$
\left.[a, b, a]=a^{p},[a, b, b]=b^{p}\right\rangle \quad(\text { for } p \geq 5) ;
$$

(x) $\left\langle a, b: a^{p}=b^{p}=[a, b]^{p}=[a, b, a]^{p}=[a, b, b]^{p}=[a, b, a, a]^{p}\right.$

$$
\begin{aligned}
=[a, b, a, b]=[a, b, b, a]=[a, b, a, a, a]= & {[a, b, a, a, b]=1, } \\
& {\left.[a, b, b, b]=[a, b, a, a]^{-m}\right\rangle }
\end{aligned}
$$

(for $p \geq 5$ and $m$ the smallest quadratic nonresidue $\bmod p$ );

(xi) $\left\langle a, b: a^{9}=b^{9}=[a, b]^{3}=[a, b, a, a]^{3}=[a, b, a, a, a]=[a, b, a, a, b]=1\right.$,

$$
\left.[a, b, a]=b^{3},[a, b, b]=a^{3},[a, b, a, a]=[a, b, b, b]\right\rangle ;
$$

(xii) $\left\langle a, b: a^{9}=b^{9}=[a, b]^{3}=[a, b, a, a]^{3}=[a, b, a, a, a]=[a, b, a, a, b]=1\right.$, $[a, b, a]^{2} \cdot[a, b, a, a]=b^{3},[a, b, b] \cdot[a, b, a, a]^{2}=a^{3}$,

$$
\langle[a, b, a, a]=[a, b, b, b]\rangle \text {. }
$$

Remarks. (1) The groups in (iv), (v) are of order $3^{5}$, those in (vi)-(ix) of order $p^{5}$, the two in (xi)-(xii) of order $3^{6}$, while the groups in $(x)$ have order $p^{6}$.

(2) All groups listed in (iv)-(vii) have their proper nonmaximal subgroups Abelian and have been characterized as $(M I)$-groups with this property in [3].

(3) The groups in (i), (ii) and (viii) are relatively free, and for the groups in (ix), (x) and (xii) the automorphism group also permutes the maximal subgroups transitively. In case of (iii), (iv), (v), (vi), (vii) and (xi) the automorphism group is nontransitive on the set of the maximal subgroups.

It is suitable to break the proof of Theorem 2 into several parts. However, most of the detailed calculation will be left to the reader.

Lemma 8. Assume that $|G|=p^{n}, \operatorname{cocl}(G)=2$ and that $G$ is $(M I)$. If $\left|G: Z_{n-3}(G)\right|=p^{3}$, then $G$ is elementary Abelian of order $p^{3}$.

Proof. Let $M$ be any maximal subgroup of $G$, with $|Z(M)|=p^{k}$. Assume that $k>n-3$, so that $M$ is Abelian. If $G$ is nonabelian, then $G$ is of class 2 , that is $n=4$, hence $p^{2}=|G: Z(G)|=\left|G: Z_{n-3}(G)\right|=p^{3}$, a contradiction; thus $G$ is Abelian and obviously it is elementary of order $p^{3}$. Suppose that $k \leq n-3 ;\left|Z_{k}(G)\right|=p^{k}$ by assumption, so since $Z(M) \leq Z_{k}(G)$, we get $Z(M)=Z_{k}(G)$. This means that $Z(M)=Z(G)$ is of order $p$, contradicting Lemma 7. 
Lemma 9. Assume that $|G|=p^{n}$ and $\operatorname{cocl}(G)=2$. If $G$ is $(M I)$ and $\left|G: Z_{n-3}(G)\right|=p^{2}$, then $4 \leq n \leq 7$. For $n=4, G$ is isomorphic to the group listed in (iii) of Theorem 2.

Proof. Let $M$ be any maximal subgroup of $G$, and denote the order of $Z(M)$ by $p^{k}$. Assume firstly that $k>n-3$; then $M$ is Abelian, whence the nilpotency class of $G$ is at most 2 . It follows that $n=4$, and then $G$ can be determined easily. If $k \leq n-3$ then $Z(M) \leq Z_{k}(G)$ and $\left|Z_{k}(G): Z(M)\right| \leq p$. Let $M_{1}$ and $M_{2}$ be different maximal subgroups in $G$. As $Z\left(M_{1}\right) \cap Z\left(M_{2}\right) \leq$ $Z_{1}(G)$, we have $\left|Z_{1}(G)\right| \geq p^{k-1},\left|Z_{k}(G): Z_{1}(G)\right| \leq p^{2}$, which yields $k \leq$ 3. Suppose that $k=3$, then $p=\left|Z_{3}(G): Z_{2}(G)\right|=\left|Z_{2}(G): Z_{1}(G)\right|=$ $\mid Z_{3}(G): Z(M)$, thus $|Z(M)|=p^{3}$ implies $|Z(G)|=p^{2}$. Therefore $Z(M) / Z(G)$ $\leq Z(G / Z(G))$, that is $Z(M) \leq Z_{2}(G)$, so that $Z(M)=Z_{2}(G)$ as their orders are equal. Being valid for any $M$, this implies that $Z_{2}(G)=Z(M)=Z(G)$, a contradiction. If $k=1$, we would get $|Z(M)|=|Z(G)|=p$, contradicting Lemma 7; thus $k=2$. If $Z(M)=Z(G)$ for some-equivalently for all- $M$ (in the light of Lemma 1 ), then $G / Z(G)$ is $(M I)$ of coclass 1 , hence $|G|=|Z(G)| \cdot|G / Z(G)| \leq p^{2} \cdot p^{3}$ by Lemma 3. Assume finally that $Z(M) \neq Z(G)$. We have $\left|Z_{2}(G): Z(M)\right|=p$, and thus $G / Z_{2}(G)$ is of maximal class. Suppose that $\left|G: Z_{2}(M)\right| \leq p$; then all maximal subgroups of $\bar{G}=G / Z_{2}(G)$ are Abelian, whence $|\bar{G}| \leq p^{3}$, so that $|G| \leq p^{6}$. If $\left|G: Z_{2}(M)\right| \geq p^{2}$, then $T=Z_{2}(M)$ is independent of the choice of $M$ since $G / Z_{2}(G)$ is of maximal class, and therefore $G / T$ is $(M I)$. Moreover, $G / T$ is either trivial or of coclass 1 , thus $|G / T| \leq p^{3}$ by Lemma 3 . On the other hand, $T \leq Z_{3}(G)$ by Lemma 5 , so $|G|=|G / T| \cdot|T| \leq p^{3} \cdot\left|Z_{3}(G)\right|=p^{7}$. (Let us mention that $Z_{3}(G)=G$ would give $p^{2}=\left|G: Z_{n-3}(G)\right| \geq\left|G: Z_{2}(G)\right|$, that is $n \leq 5$ by assumption.

For the further treatment of the open cases we point out some facts which appeared in the above proof.

Corollary 2. Suppose that $|G|=p^{n}, G$ is $(M I), \operatorname{cocl}(G)=2,\left|G: Z_{n-3}(G)\right|$ $=p^{2}$. Then

(a) if $|G: M|=p$, then $Z(M)$ is elementary Abelian of order $p^{2}$; assume that $6 \leq n \leq 7$; then

(b) $|Z(G)|=p,\left|Z_{2}(G)\right|=p^{3}, \operatorname{cocl}\left(G / Z_{2}(G)\right)=1$;

(c) $Z_{2}(G)=\langle Z(M):|G: M|=p\rangle$ is elementary Abelian;

(d) $Z_{3}(G)$ is Abelian;

(e) if $n=7$ and $|G: M|=p$ then $Z_{2}(M)=Z_{3}(G)$ is elementary Abelian;

(f) if $n=6$ and $|G: M|=p$ then either $Z_{2}(M)=Z_{2}(G)$ or $M$ is of class 2. 
Proof. There is nothing to do in case (b). For (a), suppose that $Z(M)$ were cyclic; then $G$ could not possess any noncyclic Abelian normal subgroup of order $p^{2}$. Therefore $G$ would contain a cyclic maximal subgroup by [5, Satz 7.6, page 304], a contradiction. Being in $Z_{2}(G) \leq \Phi(G)$ the centres of the maximal subgroups of $G$ centralize one another, and thus $Z_{2}(G)=$ $\langle Z(M):|G: M|=p\rangle$ is elementary Abelian.

$C_{G}\left(Z_{2}(G)\right) \geq \Phi(G) \geq Z_{3}(G)$, so $Z_{3}(G)$ is Abelian as $\left|Z_{3}(G): Z_{2}(G)\right|=p$. If $n=7$ and $M$ is a maximal subgroup of $G$, then we must have equality in all estimates used in proving that $|G| \leq p^{7}$; in particular, $Z_{2}(M)=T=$ $Z_{3}(G)$. Suppose that $Z_{3}(G)$ is of type $\left(p^{2}, p, p\right)$; then $Z_{1}(G)=\left\langle x^{p}: x \in\right.$ $\left.Z_{2}(M)\right\rangle$, so that $\bar{G}=G / Z_{1}(G)$ is also $(M I)$. As $\operatorname{cocl}(\bar{G})=2$ and $|Z(\bar{G})|=$ $\left|Z_{2}(G): Z_{1}(G)\right|=p^{2}$, we have a contradiction to (b). Assume that $n=6$ and $|G: M|=p$. If $\left|G: Z_{2}(M)\right| \neq p$ then $Z_{2}(M) \leq Z_{3}(G)=\Phi(G)$, as $G / Z_{2}(G)$ is a group of maximal class, and $Z_{2}(M) \geq Z_{2}(G)$ by Lemma 1 . Suppose that $Z_{2}(M)=Z_{3}(G)$; then $\left|M: Z_{2}(M)\right|=p$, a contradiction; and thus $Z_{2}(M)=Z_{2}(G)$, by Lemma 4 .

LEMMA 10. If $G$ is a finite p-group, $G$ is $(M I)$ and $\operatorname{cocl}(G)=2$, then $|G| \leq p^{6}$.

Proof. Suppose the contrary. Then $|G|=p^{7}$ by Lemma 9 .

CASE (A). $Z_{4}(G)$ is Abelian.

As the centre of any maximal subgroup $M$ in $G$ is of index $p^{4}$ in $M$, $Z_{4}(G)$ is the unique Abelian subgroup in $M$ of index $p$, Suppose that $Z_{4}(G)$ is of type $\left(p^{2}, p, p, p\right)$; then $Z(G)=\left\langle x^{p}: x \in Z_{4}(G)\right\rangle$ and $\bar{G}=G / Z(G)$ is also $(M I)$ of coclass 2 ; that is impossible by $($ b) of Corollary 2 , as $|Z(\bar{G})|=$ $\left|Z_{2}(G): Z_{1}(G)\right|=p^{2}$, again by (b). Thus $Z_{4}=Z_{4}(G)$ is elementary Abelian by (e) of Corollary 2. Let $x \in G \backslash Z_{4}$; then $M=Z_{4} \cdot\langle x\rangle$ is a maximal subgroup of $G$. The group $M^{\prime}=\left[Z_{4}, x\right]$ is of order $\left|Z_{4}: C_{Z_{4}}(x)\right|=\left|Z_{4}: Z(M)\right|=p^{3}$. Let $Z_{4}=\left\langle a, b, c_{1}, c_{2}, z\right\rangle, Z_{3}(G)=Z_{3}=\left\langle b, c_{1}, c_{2}, z\right\rangle, Z_{2}(G)=Z_{2}=\left\langle c_{1}, c_{2}, z\right\rangle$, $Z_{1}(G)=Z_{1}=\langle z\rangle$. We can choose $x$ with $[a, x] \in Z_{3} \backslash Z_{2}$, and we can assume

(1) $[a, x]=b$ and

(2) $Z(M)=\left\langle c_{2}, z\right\rangle$ (with $M=Z_{4} \cdot\langle x\rangle$ ). By (e) of Corollary 2, we have $b \in Z_{3}=Z_{2}(M)$, hence $[b, x]=c_{2}^{\alpha} z^{\beta}$ and $\left[c_{1}, x\right]=z^{\gamma}$. As $M^{\prime}=$ $\left\langle[a, x],[b, x],\left[c_{1}, x\right]\right\rangle$ is of order $p^{3}$, we have $\alpha \gamma \not \equiv 0(\bmod p)$; therefore we can replace $c_{2}$ by $c_{2}^{\alpha} z^{\beta}$ and $z$ by $z^{\gamma}$ to assume that

(3) $[b, x]=c_{2}$,

(4) $\left[c_{1}, x\right]=z$.

Set $G=\langle x, y\rangle$; then $a^{y}=a b^{\alpha_{1}} c_{1}^{\beta_{1}} c_{2}^{y_{1}} z^{\delta_{1}}, b^{y}=b c_{1}^{\beta_{2}} c_{2}^{\gamma_{2}} z^{\delta_{2}}, c_{2}^{y}=c_{2} z^{\delta_{3}}$. As $c_{2} \in Z(M) \backslash Z(G), \delta_{3} \not \equiv 0(\bmod p)$. Now $a^{x y}=a^{y x}$ implies, by $(1)-(4)$ : 
(5) $\beta_{2} \equiv 0(\bmod p)$. Similarly, since $b^{x y}=b^{y x}$, we get

(6) $\beta_{2} \equiv \delta_{3}(\bmod p)$. Thus by $(5)$ and $(6), \delta_{3} \equiv 0(\bmod p)$, a contradiction.

CASE (B). $Z_{4}(G)$ is nonabelian. As $G / Z_{2}(G)$ is of order $p^{4}$, it has at least one Abelian maximal subgroup, that is, we have $M_{0}^{\prime} \leq Z_{2}(G)$ for some maximal subgroup $M_{0}$ in $G$. If $M_{0}^{\prime} \neq Z_{2}(G)$, then $\left|M_{0}^{\prime}\right| \leq p^{2}$, and thus $M^{\prime} \leq Z_{2}(G)$ for every maximal subgroup $M$ of $G$; in that case $\operatorname{cl}\left(G / Z_{2}(G)\right) \leq 2$, implying that $\mathrm{cl}(G) \leq 4$, a contradiction. So $M_{0}^{\prime}=Z_{2}(G)$, hence $C_{M_{0}}\left(M_{0}^{\prime}\right)=$ $C_{M_{0}}\left(Z_{2}(G)\right)=Z_{4}(G)$. As $Z_{4}(G)$ is nonabelian and $Z_{2}(G)=Z\left(Z_{4}(G)\right)$ is of index $p^{2}$ in $Z_{4}(G)$, the derived group of $Z_{4}(G)$ is of order $p$, that is $Z_{4}(G)^{\prime}=Z_{1}(G)$.

Suppose that, for all maximal subgroups $M$ of $G, C_{M}\left(M^{\prime}\right)=Z_{4}(G)$; then $\bar{G}=G / Z_{1}(G)=G / Z_{4}(G)^{\prime}=G /\left(C_{M}\left(M^{\prime}\right)\right)^{\prime}$ is $(M I)$ and of coclass 2 with $\mid Z\left(\bar{G}|=| Z_{2}(G): Z_{1}(G) \mid=p^{2}\right.$, contradicting (b) of Corollary 2. Thus there exists some maximal subgroup $M_{1}$ in $G$ with $C_{M_{1}}\left(M_{1}^{\prime}\right) \neq C_{M_{0}}\left(M_{0}^{\prime}\right)$. As by (e) of Corollary 2 we have $Z_{3}(G)=Z_{2}\left(M_{i}\right) \leq C_{M_{i}}(i=1,2)$, we get

$$
G^{\prime} \leq C_{M_{0}}\left(M_{0}^{\prime}\right) \cap C_{M_{1}}\left(M_{1}^{\prime}\right)=Z_{3}(G)
$$

since $\left|G: C_{M_{i}}\left(M_{i}^{\prime}\right)\right|=p^{2}$, and this is the final contradiction.

LEMMA 11. Assume that $|G|=p^{5}, \operatorname{cocl}(G)=2$. If $G$ is $(M I)$, then $Z_{2}(G)$ is elementary Abelian of order $p^{3}$ and $Z_{1}(G)$ is of order $p^{2}$.

Proof. Clearly $Z_{2}(G)=\Phi(G)$ is noncyclic Abelian of order $p^{3}$ by Lemmas 1,2 and (a) of Corollary 2. Suppose that $\left|Z_{1}(G)\right|=p$. For any maximal subgroups $M_{1}, M_{2}$ of $G, G / Z\left(M_{1}\right)$ is of order $p^{3}$, and hence $M_{2}^{\prime} \leq Z\left(M_{1}\right)$. Taking $M_{1} \neq M_{2}$ we get $M_{2}^{\prime} \leq Z\left(M_{1}\right) \cap Z\left(M_{2}\right)=Z(G)$, that is $M^{\prime}=Z(G)$ for all maximal subgroups $M$. The group $G / Z(G)$ is therefore $(M I)$ and one step nonabelian of order $p^{4}$, so that $G / Z(G)=\left\langle\bar{x}, \bar{y}: \bar{x}^{p^{2}}=\bar{y}^{p^{2}}=1, \bar{y}^{-1} \overline{x y}=\right.$ $\left.\bar{x}^{1+p}\right\rangle$. Obviously $G=\langle x, y\rangle(\bar{x}=x Z(G), \bar{y}=y Z(G))$ is not metacyclic, hence $x^{y}=x^{1+p} c$ where $\langle c\rangle=Z(G)$. For any integer $n,\left(x^{n}\right)^{y}=x^{n(1+p)} c^{n}$; in particular, $\left[x^{p}, y\right]=x^{p^{2}}$. Suppose that $x^{p^{2}} \neq 1$; then $\left\langle x^{p^{2}}\right\rangle=Z(G),\langle x\rangle \triangleleft G$, $G$ is metacyclic, a contradiction; thus the order of $x$ is $p^{2}$. For any integer $n$ we have

$$
x^{y^{n}}=x^{(1+p)^{n}} c^{\left((1+p)^{n}-1\right) / p} .
$$

In particular $\left[x, y^{p}\right]=1$; that implies $x^{p}, y^{p} \in Z(G)$, whence $p=|Z(G)| \geq$ $\left|\left\langle x^{p}, y^{p}\right\rangle\right| \geq\left|\left\langle\bar{x}^{p}, \bar{y}^{p}\right\rangle\right|=p^{2}$, a contradiction.

Suppose that $Z_{2}(G)$ is not elementary. Then by earlier remarks, it is Abelian of type $\left(p^{2}, p\right)$. Since $|Z(G)|=p^{2}$, we have $Z(G)=Z(M)$ for every maximal subgroup $M$ of $G$ by Lemma 1 and (a) of Corollary 2; $G / Z(G)$ is therefore $(M I)$ of order $p^{3}$. As $G / Z(G)$ is never quaternion, $p>2$ and $G / Z(G)$ is of exponent $p$, that is $\mho(G) \leq Z(G)$. Suppose that $|\mho(G)|=p$. 
Then $\mho(G)=\mho(M)$ for all maximal subgroups $M$; hence $\bar{G}=G / \mho(G)$ is $(M I)$ of order $p^{4}$ and of exponent $p$. The class of $\bar{G}$ must be 3 since $\bar{G}$ is 2 generated; but there is no $(M I)$-group of maximal class and of order $p^{4}$. Thus $\mho(G)=Z(G)$, and hence we can find an element $g$ in $G$ with $g^{p} \notin \mho\left(Z_{2}(G)\right.$ ). Let $K=\langle g\rangle \cdot Z_{2}(G)$, so that $K$ is a maximal subgroup in $G$ and $(K)$ is of order (at least) $p^{2}$. This implies that the order of $\Phi(K)$ is (at least) $p^{2}$; as $\Phi(K)=\mho(K)=\mho(G)=Z(G)=Z(K)$, all maximal subgroups of $K$ are Abelian, hence $G$ is isomorphic to one of the groups listed in (iv)-(vii), by [2]. On the other hand, all the above groups have their Frattini subgroup elementary Abelian, a contradiction.

Proposition 1. Assume that $|G|=p^{5}$ and $\operatorname{cocl}(G)=2$. If $G$ is $(M I)$, then $G$ is isomorphic to one of the groups listed in (iv)-(ix).

Proof. We have $G=\langle x, y\rangle, Z_{2}:=Z_{2}(G)=\left\langle a, z_{1}, z_{2}\right\rangle=\Phi(G)$ is elementary Abelian, where $a=[x, y]$ and $Z_{1}:=\left\langle z_{1}, z_{2}\right\rangle=Z(G)=Z(M)$ for all maximal subgroups $M$. By $(M I)$, either $\Omega(G)=G$ or $\Omega(G) \leq \Phi(G)$; thus we can assume that the elements $x$ and $y$ have the same order $p$ or $p^{2}$. Since the maximal subgroups of $G$ are nonabelian, $Z_{2}=C_{G}\left(Z_{2}\right)=C_{G}(a)$. Therefore $\langle[a, x],[a, y]\rangle=[a, G]$ is of order $p^{2}$, that is, one can assume that $a^{x}=a z_{1}, a^{y}=a z_{2}$. Suppose that $p=2$, when $\left[x, y^{2}\right]=x^{-1}(x a)^{y}=z_{2}$, and consequently $y^{2} \in Z_{2} \backslash Z_{1}$. Thus we have $y^{2}=a c$ (with $c \in Z_{1}$ ), and hence $z_{2}=\left[x, y^{2}\right]=[x, a]=z_{1}$, a contradiction; so $p>2$. For any integer $n, x^{y_{n}}=x a^{n} z_{2}^{\left(\begin{array}{l}n \\ 2\end{array}\right)}$; this implies that $y^{p} \in Z(G)$, and similarly $x^{p} \in Z(G)$.

CASE 1. $x^{p}=y^{p}=1$.

The generators $x, y$ obviously satisfy the relations of the presentation in (viii) of Theorem 2. That relations yield that $G$ is $(M I)$ if and only if $p \geq 5$.

CASE 2. $x$ and $y$ are of order $p^{2}$.

Let $M$ denote a maximal subgroup of $G$. If $\Phi(M)$ is of order $p^{2}$, then $\Phi(M)=Z(M)$, that is $M$ is a one-step nonabelian group. That means that $G$ is exactly one of the groups listed in (iv)-(vii), by [3]. Therefore we can assume $|\Phi(M)|=p$, equivalently $\mho(M)=M^{\prime}$; in particular, $x^{p}=z_{1}^{\alpha}$, $y^{p}=z_{2}^{\beta}$. For $p=3$ we get a contradiction; for $p \geq 5 \alpha=\beta$ can be assumed to be 1 , that is $G$ is the group in (ix), which in turn is $(M I)$.

LEMma 12. Assume that $G$ is $(M I)$ of order $p^{6}$ and that $\operatorname{cocl}(G)=2$; then $Z_{3}(G)$ is elementary Abelian, and every maximal subgroup in $G$ is of class 3 .

Proof. Suppose that all maximal subgroups of $G$ are of class 2. Suppose that $G=\langle x, y\rangle$ and $[x, y]=a$. Since $Z_{3}(G)$ is Abelian by (d) of Corollary $2, x^{p} \in Z\left(\left\langle Z_{3}(G), x\right\rangle\right)$. As $\left\langle Z_{3}(G), x\right\rangle$ is a maximal subgroup of $G, x^{p}$ lies in 
$Z_{2}(G)$ by (a); hence $\mho(G) \leq Z_{2}(G)$. It follows that $G / Z_{2}(G)$ is a nonabelian group of exponent $p$, so $p$ must be odd.

Since every maximal subgroup of $G$ is supposed to be of class 2 , we have $[a, x]=c \in C_{G}(x),[a, y]=d \in C_{G}(y), c^{y}=c z^{\alpha}, d^{x}=d z^{\beta}$ (with $Z(G)=$ $\langle z\rangle)$. Since $Z_{3}(G)=\left\langle Z_{2}(G), a\right\rangle$, we have $Z_{2}(G)=Z\left(Z_{3}(G) \cdot\langle x\rangle\right) \cdot Z\left(Z_{3}(G)\right.$. $\langle y\rangle)=\left(Z_{3}(G) \cdot\langle x\rangle\right)^{\prime} \cdot\left(Z_{3}(G) \cdot\langle y\rangle\right)^{\prime}=\langle c, d, Z(G)\rangle$, and therefore $\alpha \neq 0 \neq \beta$. Set $K=Z_{3} \cdot\langle x y\rangle$. Then equations $[a, x y]=a^{-1}(a c)^{y}=d c z^{\alpha}$ and $[a, y x]=$ $a^{-1}(a d)^{x}=c d x^{\beta}$ imply that $\alpha=\beta$, as $Z_{3}(G)$ is Abelian. Since $K^{\prime} \leq Z(K)$, we can deduce that $1=[c d, x y]=z^{2 \alpha}$. Thus $\alpha=0$, a contradiction; it proves that the maximal subgroups of $G$ are of nilpotency class 3 , by (f).

Suppose that $Z_{3}(G)$ is (Abelian) of type $\left(p^{2}, p, p\right)$; then $Z_{3}(G)=\left\langle g, b_{1}, b_{2}\right\rangle$, $Z_{2}(G)=\left\langle b_{1}, b_{2}, g^{p}\right\rangle, Z_{1}(G)=\left\langle g^{p}\right\rangle$. Let $M_{1}=\left\langle Z_{3}(G), x\right\rangle$ be a maximal subgroup of $G$ with $Z\left(M_{1}\right)=\left\langle b_{1}, g^{p}\right\rangle$; then by choosing $b_{2}$ suitably we can assume that $[g, x]=b_{2},\left[b_{1}, x\right]=1,\left[b_{2}, x\right]=g^{\alpha p},(\alpha \neq 0)$ : Since the order of $M_{1}^{\prime}$ is $p^{2}$ we can find a maximal subgroup $M_{2}=\left\langle Z_{3}(G), y\right\rangle$ such that $Z\left(M_{2}\right)=$ $M_{1}^{\prime}=\left\langle b_{2}, g^{p}\right\rangle$. Obviously $[g, y]=b_{1}^{\gamma} b_{2}^{\delta} \cdot g^{\varphi p},\left[b_{1}, y\right]=g^{\beta p},\left[b_{2}, y\right]=1(\gamma \neq 0)$; hence $g^{x y}=\left(g b_{2}\right)^{y}=g b_{1}^{\gamma} \cdot b_{2}^{1+\delta} \cdot g^{\varphi p}, g^{y x}=\left(g b_{1}^{\gamma} \cdot b_{2}^{\delta} \cdot g^{\varphi p}\right)^{x}=\left(g b_{2}\right) b_{1}^{\gamma}$. $\left(b_{2} g^{\alpha p}\right)^{\delta} \cdot g^{\varphi p}=g b_{1}^{\gamma} \cdot b_{2}^{1+\delta} \cdot g^{(\varphi+\alpha \delta) p}$. Since $g^{x y}=g^{y x}$, we have $\alpha \delta=0$, and thus $\delta=0$ (since $\alpha \neq 0)$. Replacing $b_{1}$ by $b_{1}^{\gamma} \cdot g^{\varphi p}$ one can assume $[g, y]=b_{1}$, $\left[b_{1}, y\right]=g^{\beta p},\left[b_{2}, y\right]=1$ (with a new value for $\beta$ ).

We show that the quadratic character of $\alpha(\bmod p)$ is an invariant of $M_{1}$. For, let $g^{\prime}=g^{u} b_{1}^{v} b_{2}^{t}, x^{\prime}=x^{i} s\left(s \in Z_{3}(G)\right)$, in which case

$$
\begin{aligned}
& b_{2}^{\prime}:=\left[g^{\prime}, x^{\prime}\right]=\left[g, x^{i}\right]^{u} \cdot g^{\alpha t i p} \\
& \quad=\left(b_{2}^{i} g^{(i) \alpha p}\right)^{u} g^{\alpha t i p}=b_{2}^{i u} g^{\left(u\left(\frac{i}{2}\right)+t i\right) \alpha p}, \\
& {\left[b_{2}^{\prime}, x^{\prime}\right]=g^{i^{2} u \alpha p}=\left(g^{\prime p}\right)^{i^{2} \alpha} .}
\end{aligned}
$$

Now for $\{k, m\} \neq\{0,0\}$, set $M_{k m}=\left\langle Z_{3}(G), x^{k} y^{m}\right\rangle$; the invariant of $M_{k m}$ is $\alpha k^{2}+\beta m^{2}$, as

$$
\begin{aligned}
& {\left[b, x^{k} y^{m}\right]=b_{2}^{k} b_{1}^{m} \quad(\bmod Z(G))} \\
& {\left[g, x^{k} y^{m}, x^{k} y^{m}\right]=\left[b_{1}^{m} b_{2}^{k}, x^{k} y^{m}\right]=g^{p\left(\alpha k^{2}+\beta m^{2}\right)}}
\end{aligned}
$$

Now $G$ can be $(M I)$ only if $\alpha, \beta, \alpha k^{2}+\beta m^{2}$ have the same quadratic character $(\bmod p)$ for all nonzero pairs $\{k, m\}$. However, this does not happen for any prime $p$, and we have a contradiction. Thus $Z_{3}(G)$ is elementary Abelian by (c) of Corollary 2.

Using the previous lemma we can finish the proof of Theorem 2 by a direct calculation to get

Proposition 2. Assume that $G$ is $(M I)$ of order $p^{6}$ and $\operatorname{cocl}(G)=2$; then $G$ is isomorphic to one of the groups listed in (x)-(xii). 
Before turning to the case of coclass 3 , we prove

LEMMA 13. If the finite p-group $G$ is $(M I)$ and has a maximal subgroup $M$ with $Z(M) \$ \Phi(G)$, then the nilpotency class of $G$ is at most 2 .

Proof. There exists a maximal subgroup $M_{1}$ in $G$ with $Z(M) \$ M_{1}$, that is, $G=Z(M) \cdot M_{1}$. Then $\left.\left[Z\left(M_{1}\right), G\right]=Z\left(M_{1}\right), Z(M)\right] \leq Z\left(M_{1}\right) \cap Z(M)=$ $Z(G)$, and thus $Z\left(M_{1}\right) \leq Z_{2}(G)$. Suppose that the class of $G$ is greater than 2, then by Lemma $1 Z\left(M_{1}\right) \leq Z_{2}(G) \leq \Phi(G)$. Thus $Z\left(M_{1}\right) \leq M \leq C_{G}(Z(M))$, hence $Z(M) \leq C_{G}\left(Z\left(M_{1}\right)\right)=M_{1}$, a contradiction.

Now our aim is to prove

Theorem 3. If $G$ is a finite p-group, $G$ is $(M I)$ and the coclass of $G$ is 3 , then $|G| \leq p^{13}$.

We will get Theorem 3 as a corollary to three propositions.

Proposition 3. Assume that $G$ is $(M I),|G|=p^{n}, \operatorname{cl}(G)=n-3$ and $\left|G: Z_{n-4}(G)\right|=p^{4}$; then either

(i) $G$ is Abelian of order $p^{4}$, or

(ii) $G$ is extraspecial of order $p^{5}$.

Proof. Let $M$ be a maximal subgroup of $G$, and denote the order of $Z(M)$ by $p^{k}$.

CASE 1. $|G: Z(M)|=p$.

Either $G$ is Abelian, giving (i) or $G$ is one step nonabelian. In the latter case $2=\operatorname{cl}(G)=n-3$, so that $n=5$, which yields that $p^{2}=|G: Z(G)|=$ $\left|G: Z_{1}(G)\right|=\left|G: Z_{n-4}(G)\right|=p^{4}$, a contradiction.

CASE 2. $|G: Z(M)|=p^{3}$.

Suppose that $Z(M) \leq \Phi(G)=\Phi$ for all maximal subgroups $M$. The equality $Z(M)=\Phi$ would imply that $Z(M)=Z(G)$, hence $\left|G: Z_{1}(G)\right|=p^{3}$, a contradiction; thus $|\Phi: Z(M)|=p$ and $Z(M)>Z(G)$. So, with different maximal subgroups $M_{1}$ and $M_{2},\left|\Phi: Z_{1}(G)\right|=\left|\Phi: Z\left(M_{1}\right) \cap Z\left(M_{2}\right)\right|=p^{2}$ gives $|Z(M): Z(G)|=p$, so that $\left|G: Z_{1}(G)\right|=p^{4}, n=5,\left|Z_{1}(G)\right|=p$, $\operatorname{cl}(G)=2$. Since $G$ is 2-generator, say $G=\langle x, y\rangle$, and $\left|G^{\prime}\right|=p,\left\langle x^{p}, y, Z(G)\right\rangle$ is an Abelian maximal subgroup of $G$, a contradiction; therefore $Z\left(M_{0}\right) \$ \Phi$ for some maximal subgroup $M_{0}$, whence $\operatorname{cl}(G)=2$ by Lemma 13 . We have then $n=5,\left|G^{\prime}\right|=p, M^{\prime}=G^{\prime}$; hence $G / G^{\prime}$ is also $(M I)$. If it were of type $\left(p^{2}, p^{2}\right)$, then we would get the same contradiction as above. Thus $G / G^{\prime}$ is elementary Abelian, that is $G$ is extraspecial.

CASE 3. $|G: Z(M)| \geq p^{4}$. 
$|Z(M)|=p^{k}$ implies that $Z(M) \leq Z_{k}(G) \leq Z_{n-4}(G)$, so that $Z(M)=$ $Z_{i}(G)$ for some $i$, by Lemma 4. As $i$ is independent of $M$, that gives $Z(M)=$ $Z(G)$, and thus $\bar{G}=G / Z(G)$ is also $(M I)$ of coclass 3. By the induction hypothesis, either

(i) $\bar{G}$ is Abelian of order $p^{4}$, or

(ii) $\bar{G}$ is extraspecial of order $p^{5}$.

In case $(\mathrm{i}), \operatorname{cl}(G)=2, n=5$, whence $\left|G: Z_{1}(G)\right|=p^{4}=|G: Z(M)|$; that is $Z(G)=Z(M)$ is of order $p$, contradicting Corollary 2 . In case (ii), $\operatorname{cl}(G)=3$, $n=6$, so $p^{4}=\left|G: Z_{2}(G)\right|$. This implies that $Z(M) \leq Z_{2}(G)$, but then $Z(M)=Z_{2}(G)$, as $p^{2} \leq|Z(M)| \leq\left|Z_{2}(G)\right|=p^{2}$. Since $Z\left(M_{1}\right)=Z\left(M_{2}\right)$ for any maximal subgroups $M_{1}, M_{2}$, we have $Z_{2}(G)=Z(M)=Z_{1}(G)$, a contradiction.

LEMMA 14. Suppose that $|G|=p^{n}, \operatorname{cl}(G)=n-3$ and $\left|Z_{2}(G)\right|=p^{3}=$ $\left|G: Z_{n-4}(G)\right|$. Assume that $Z_{2}(M) \geq Z_{2}(G) \geq Z(M)$ and $\left|Z_{2}(M)\right| \geq p^{n-3}$ for all maximal subgroups $M$ of $G$; then $n \leq 8$.

Proof. $Z_{2}(M) \geq \gamma_{3}(G)$, as $\operatorname{cl}\left(G / Z_{2}(M)\right) \leq 2$. Since $\operatorname{cl}(G)=n-3$, we have $\gamma_{3}(G) \leq Z_{n-5}(G) \leq Z_{n-4}(G)$, and hence there exists some $i$ with $\gamma_{3}(G) Z_{2}(G)=Z_{i}(G)$, by Lemma 4 . Certainly $i=n-5$, and thus $Z_{2}(M) \geq$ $\gamma_{3}(G) Z_{2}(G)=Z_{n-5}(G)$. Let $M_{1} \neq M_{2}$ be maximal subgroups in $G$, so that, by Lemma $5, Z_{n-5}(G) \leq Z_{2}\left(M_{1}\right) \cap Z_{2}\left(M_{2}\right) \leq Z_{3}(G)$, so $n-5 \leq 3$.

Proposition 4. If $G$ is $(M I),|G|=p^{n}, \mathrm{cl}(G)=n-3$ and $\left|G: Z_{n-4}(G)\right|=$ $p^{3}$, then $n \leq 8$.

Proof. Let $M$ be any maximal subgroup of $G$ and $|Z(M)|=p^{k}$.

CASE 1 . $|G: Z(M)|=p$.

$G$ is either Abelian of order $p^{4}$ or one step nonabelian of order $p^{5}$. In the first case $p^{3}=\left|G: Z_{n-4}(G)\right|=\left|G: Z_{0}(G)\right|=p^{4}$, a contradiction. If $n=5$, then $p^{3}=\left|G: Z_{n-4}(G)\right|=\left|G: Z_{1}(G)\right|=p^{2}$, also a contradiction.

CASE 2. $|G: Z(M)|=p^{3}$.

If $Z(M)=\Phi(G)=\Phi$ for all $M$, then $Z(M)=\Phi=Z(G)$ is of index $p^{3}$ in $G$, and therefore $n=5$. If $|\Phi: Z(M)|=p$ for all $M$, then with $M_{1} \neq M_{2}$ (maximal subgroups in $\left.G\right),\left|\Phi: Z_{1}(G)\right| \leq\left|\Phi: Z\left(M_{1}\right) \cap Z\left(M_{2}\right)\right| \leq p^{2}$, hence $\left|G: Z_{1}(G)\right| \leq p^{4}$ and $n-4 \leq 2$. If $Z\left(M_{0}\right) \not \Phi$ for some $M_{0}$, then $n-3=\operatorname{cl}(G) \leq 2$, by Lemma 13 .

CASE 3. $|G: Z(M)| \geq p^{4}$.

$Z(M) \leq Z_{k}(G) \leq Z_{n-4}(G)$, so since $\operatorname{cocl}(G)=3$ and $\left|G: Z_{n-4}(G)\right|=$ $p^{3}$, we have $\left|Z_{k}(G): Z(M)\right|=p$. Were $Z_{k}(G)=Z(M)$, one would get $k=1$, contrary to Lemma 7 ; thus $\left|Z_{k}(G): Z(M)\right|=p$. Suppose that 
$Z(M)=Z_{1}(G) ;$ then $k=2$, and $\bar{G}=G / Z_{1}(G)$ is $(M I)$ with $\operatorname{cocl}(\bar{G})=2$ and $\left|\bar{G}: Z_{n-5}(\bar{G})\right|=p^{3}$. Now Lemma 8 says that $|\bar{G}|=p^{3}$, so that $|G| \leq p^{5}$. Assume from now on that $Z(M)>Z(G)$; for any $M_{1} \neq M_{2}$ (maximal subgroups in $G),\left|Z_{k}(G): Z_{1}(G)\right|=\left|Z_{k}(G): Z\left(M_{1}\right) \cap Z\left(M_{2}\right)\right|=p^{2}$, so that $2 \leq k \leq 3$. The inequalities $Z_{k}(G) \geq Z(M) \geq Z_{1}(G)$ exclude the possibility of $k=3$ by Lemma 4 , and hence $k=2$. Therefore $\left|Z_{2}(G): Z(M)\right|=$ $p=\left|Z(M): Z_{1}(G)\right|$, and $\left|Z_{1}(G)\right|=p$. If $\left|Z_{2}(M)\right| \geq p^{n-3}$, then $|G| \leq p^{8}$ by Lemma 14. Thus $\left|Z_{2}(M)\right| \leq p^{n-4}$, whence $Z_{2}(G) \leq Z_{2}(M) \leq Z_{n-4}(G)$, so that $Z_{2}(M)=Z_{i}(G)$ for some $2 \leq i \leq n-4$ by Lemma 4 . As $Z(M) \leq Z_{2}(G)$, Lemma 5 gives $i \leq 3$. On the other hand, $\bar{G}=G / Z_{i}(G)$ is $(M I)$ of coclass 2 and $\left|\bar{G}: Z_{n-i-4}(\bar{G})\right|=p^{3}$, thus $|\bar{G}|=p^{3}$, consequently $|G|=p^{3} \cdot\left|Z_{i}(G)\right|=$ $p^{3+i+1} \leq p^{7}$.

Proposition 5. If $G$ is $(M I),|G|=p^{n}, \operatorname{cl}(G)=n-3$ and $\left|G: Z_{n-4}(G)\right|=$ $p^{2}$, then $n \leq 13$.

Proof. Let $M$ be any maximal subgroup in $G$ and $|Z(M)|=p^{k}$; we can assume that $Z_{k}(G)<G$. In the same way as earlier, we may assume that $|G: Z(M)| \geq p^{3}$, as well as $Z(M) \leq \Phi(G)=Z_{n-4}(G)$. We define

$$
t(M)=\min \left\{i: Z(M) \leq Z_{i}(G)\right\} .
$$

Assume that $t\left(M_{0}\right)=1$ for some $M_{0}$. Then $t(M)=1$ for any $M$ by Lemma 1. Now $\bar{G}=G / Z(G)$ is $(M I), \operatorname{cocl}(\bar{G}) \leq 2$ (by Lemma 7), and thus $|\bar{G}| \leq p^{6}$ by Theorem 2 and Lemma 3 ; so $|G| \leq p^{6}|Z(G)|=p^{6+k} \leq p^{9}$. We can therefore assume that $t(M)>1$ for every $M$; if $M_{1}$ and $M_{2}$ are different maximal subgroups in $G$, then

(E) $\left|Z_{k}(G): Z_{1}(G)\right|=\left|Z_{k}(G): Z\left(M_{1}\right) \cap Z\left(M_{2}\right)\right| \leq\left|Z_{k}(G): Z(M)\right|^{2} \leq p^{4}$

(for any $M$ ), whence $2 \leq t(M) \leq k \leq 5$.

Suppose that $t\left(M_{0}\right)=5$ for some $M_{0}$, then $k=5$, so we have equalities in (E). For any $M, Z_{1}(G) \leq Z(M) \leq Z_{5}(G)$ implies that $Z(M)=Z_{i}(G)$ for some $i$, by Lemma 4; necessarily $i=1$, a contradiction; thus we may assume that $2 \leq t(M) \leq 4$ for all $M$. Suppose that $t\left(M_{0}\right)=4$ for some $M_{0}$. We have $\left|Z_{4}(G): Z_{1}(G)\right| \leq p^{4}$ by $(E)$; if equality holds here, then $k=4$, and by the second part of $(E)$, it follows that $\left|Z_{4}(G): Z(M)\right|=p^{2}$, whence $\left|Z(M): Z_{1}(G)\right|=p^{2}$. The latter implies that $Z(M) / Z_{1}(G) \leq Z_{2}\left(G / Z_{1}(G)\right)$, that is $Z(M) \leq Z_{3}(G)$; particularly $Z\left(M_{0}\right) \leq Z_{3}(G)$, a contradiction. So $\left|Z_{4}(G): Z_{1}(G)\right|=p^{3}$, hence $Z_{4}(G) \geq Z(M) \geq Z_{1}(G)$ leads to $Z(M)=Z_{i}(G)$ for some $i$ by Lemma 4 . Clearly $i=1$, that is $t(M)=1$, a contradiction; thus we may assume that $2 \leq t(M) \leq 3$. In particular, $Z(M) \leq Z_{3}(G)$ for all $M$. 
Suppose that $Z_{2}\left(M_{0}\right) \nless Z_{n-4}(G)$ for some $M_{0}$; then $G=Z_{2}\left(M_{0}\right) \cdot M_{1}$, with a suitable maximal subgroup $M_{1} \neq M_{0}$. So $\left[Z_{2}\left(M_{1}\right), G\right] \leq Z\left(M_{1}\right)$. $\left(Z_{2}\left(M_{1}\right) \cap Z_{2}\left(M_{0}\right)\right) \leq Z_{4}(G)$ by Lemma 5 , thus $Z_{2}\left(M_{1}\right) \leq Z_{5}(G)$. We have $\left|Z_{2}(M)\right|=\left|Z_{2}\left(M_{1}\right)\right| \leq\left|Z_{5}(G)\right| \leq p^{7}$ (or $n \leq 8$ ) for all maximal subgroups $M$. As $Z_{2}\left(M_{0}\right) \leq Z_{7}(G) \not Z_{n-4}(G)$, we get that $n-4<7, n \leq 10$.

Assume now that $Z_{2}(M) \leq Z_{n-4}(G)$ for all maximal subgroups $M$. Let $s(\leq n-4)$ be chosen to be minimal, subject to $Z_{2}(M) \leq Z_{s}(G)$ for all $M$.

Claim. $\left|Z_{s}(G): Z_{2}(M)\right| \leq p$ (for all $M$ ).

First, $\left|Z_{2}(G): Z_{1}(G)\right| \geq p^{2}$. In fact, supposing that $\left|Z_{2}(G): Z_{1}(G)\right|=p$, we can find $N_{i} \triangleleft G$ with $Z\left(M_{i}\right) \geq N_{i} \geq Z(G),\left|N_{i}: Z_{1}(G)\right|=p$ (for all maximal subgroups $\left.M_{i}\right)$. Then $N_{i} / Z_{1}(G) \leq Z\left(G / Z_{1}(G)\right)$, that is $N_{i} \leq Z_{2}(G)$; so $Z_{2}(G)=N_{i} \leq Z\left(M_{i}\right)$ for all $i$, resulting in $Z_{2}(G) \leq \bigcap_{i} Z\left(M_{i}\right)=Z_{1}(G)$, a contradiction. Therefore $\operatorname{cocl}\left(G / Z_{2}(G)\right) \leq 2$, which proves our claim.

For any $M_{1} \neq M_{2}$ (maximal subgroups of $G$ ),

$$
p^{2} \geq\left|Z_{s}(G): Z_{2}\left(M_{1}\right) \cap Z_{2}\left(M_{2}\right)\right| \geq\left|Z_{s}(G): Z_{4}(G)\right|
$$

(by Lemma 5); that shows $s \leq 6$. If $Z_{s}(G)=Z_{2}(M)$ (for all $M$ ) then $G / Z_{s}(G)$ is $(M I)$ of coclass $c \leq 2$, so

$$
|G|=\left|G / Z_{s}(G)\right| \cdot\left|Z_{s}(G)\right| \leq p^{3 c} \cdot p^{s+(3-c)} \leq p^{13}
$$

by Theorem 2 and Lemma 3 . In the remaining case $\left|Z_{s}(G): Z_{2}(M)\right|=p$ (for all $M$ ), thus $\left|Z_{s}(G): Z_{2}(G)\right|=p^{s-2}$ cannot hold by Lemma 4 . Hence $G / Z_{s}(G)$ is of maximal class (or it is trivial, yielding $|G|=\left|Z_{s}(G)\right| \leq p^{s+3} \leq p^{9}$ ). We have $Z_{3}(M) \geq Z_{s}(G)$ (for all $M$ ) since $\left|Z_{s}(G): Z_{2}(M)\right|=p$; on the other hand, $Z_{3}(M) \leq Z_{s+5}(G) \leq Z_{11}(G)$ by Lemma 6 , whence $\left|Z_{3}(M)\right| \leq\left|Z_{11}(G)\right|$, so that $\left|Z_{3}(M)\right| \leq p^{13}$. Now either $\left|Z_{3}(M)\right|=p^{n-1}$, whence $\mathrm{cl}(M) \leq 3$, $\operatorname{cl}(G) \leq 6, n \leq 9$, or $Z_{3}(M)=Z_{i}(G)$ for some $s \leq i \leq s+5$ by Lemma 4. As for that latter case, $G / Z_{i}(G)$ is $(M I)$ of coclass $\leq 1$, and we can use Lemma 5 to conclude that $\left|Z_{i}(G)\right|=\left|Z_{3}(M)\right| \leq\left|Z_{s+1}(G)\right| \leq\left|Z_{7}(G)\right|$, involving $\left|Z_{i}(G)\right| \leq p^{9}$. We can summarize our conclusions as follows:

$$
|G|=\left|G / Z_{i}(G)\right| \cdot\left|Z_{i}(G)\right| \leq p^{3} \cdot p^{9}=p^{12} .
$$

\section{References}

[1] R. M. Bryant and L. C. Kovács, 'Lie representations and groups of prime-power order', $J$. London Math. Soc. (2) 17 (1987), 415-421.

[2] P. Hall, 'A contribution to the theory of groups of prime power orders', Proc. London Math. Soc. 36 (1933), 29-95.

[3] P. Z. Hermann, 'On a class of finite groups having isomorphic maximal subgroups', Ann. Univ. Sci. Budapest. Eötvös, Sect. Math. 24 (1981), 87-92. 
[4] G. Higman, 'Suzuki 2-groups', Illinois J. Math. 7 (1963), 79-96.

[5] B. Huppert, Endliche Gruppen I, (Springer, Berlin-Heidelberg-New York, 1967).

\section{Department of Algebra and Number Theory}

Eötvös Loránd University

Múzeum krt. 6-8

H-1088 Budapest

Hungary 